\title{
Prevalência e fatores de risco associados à infecção por Toxoplasma gondii em granjas suinícolas tecnificadas no Estado de Alagoas ${ }^{1}$
}

\author{
Rômulo Menna B. Valença ${ }^{2}$, Rinaldo A. Mota ${ }^{3}$, Giulliano A. Anderlini², \\ Eduardo B. de Faria ${ }^{3}$, Érica F.S.T.F. Cavalcanti ${ }^{3}$, Pedro Paulo F. \\ Albuquerque $^{3}$, Orestes Luiz de S. Neto ${ }^{3}$ e Maria Madalena P. Guerra ${ }^{3}$
}

\begin{abstract}
Valença R.M.B., Mota R.A., Anderlini G.A., Faria E.B., Cavalcanti E.F.S.T.F., Albuquerque P.P.F., Neto O.L.S. \& Guerra M.M.P. 2011. [Prevalence and risk factors associated with infection by Toxoplasma gondii on commercial swine farms in the state of Alagoas, Brazil.] Prevalência e fatores de risco associados à infecção por Toxoplasma gondii em granjas suinícolas tecnificadas no Estado de Alagoas. Pesquisa Veterinária Brasileira 31(2):121-126. Curso de Medicina Veterinária, Centro Universitário Cesmac, Rodovia Divaldo Suruagy s/n, Quadra 04, Lote 04, Marechal Deodoro, AL 57160-000, Brazil. E-mail: rmbvalenca@ig.com.br

This study was aimed to determine the prevalence and to identify risk factors associated with infection by Toxoplasma gondii in pigs from commercial swine farms in the state of Alagoas, Brazil. To compose the sample size of prevalence 342 pigs were used, with 312 sows and 30 boars, from of seven swine farms and distributed in five districts of the state of Alagoas. The serological examination for the detection of anti-Toxoplasma gondii antibodies was achieved by Indirect Immunofluorescence (IFAT), using anti-pig IgG-conjugated to fluorescein isothiocyanate. The analysis of risk factors were performed by the application of questionnaires consisting of objective questions relating to the designer, the general characteristics of the property, the production, reproductive and health management. The prevalence was of $26,9 \%$ (92/342) of seropositive pigs. The associated factor was the boars introduction on farms in the last five years ( $p=0,014 ; \mathrm{OR}=1,83 ; \mathrm{Cl}=1,13-2,96)$. It is concluded that infection with Toxoplasma gondii is widespread in commercial pig farms in the state of Alagoas, Brazil. The results suggest the control of cat population, the performance of cleaning and sanitation practices and laboratory testing to detect of infection by $T$. gondii in animals to be placed on the farms as measures to reduce the infection rates on region studied.
\end{abstract}

INDEX TERMS: Toxoplasma gondii infection, swine.

RESUMO.- Objetivou-se com este estudo calcular a prevalência e identificar os fatores de risco associados à infecção por Toxoplasma gondii em suínos criados em granjas tecnificadas no Estado de Alagoas, Brasil. Para compor a amostra do estudo de prevalência foram utilizados

\footnotetext{
${ }^{1}$ Recebido em 16 de abril de 2010.

Aceito para publicação em 14 de setembro de 2010.

Parte da tese de doutorado em Medicina Veterinária do primeiro autor, Universidade Federal Rural de Pernambuco (UFRPE).

${ }^{2}$ Curso de Medicina Veterinária, Centro Universitário Cesmac, Rodovia Divaldo Suruagy s/n, Quadra 04, Lote 04,, Marechal Deodoro, AL 57160-000, Brasil. *Autor para correspondência: rmbvalenca@ig.com.br

${ }^{3}$ Departamento de Medicina Veterinária, UFRPE, Av. Dom Manoel de Medeiros s/n, Recife, PE 52171-900, Brasil.
}

342 suínos, sendo 312 matrizes e 30 varrões, oriundos de sete granjas de ciclo completo e distribuídas em cinco municípios do Estado de Alagoas. O exame sorológico para a pesquisa de anticorpos anti-Toxoplasma gondii foi realizado através da técnica de Imunofluorescência Indireta (RIFI), utilizando-se anticorpos anti-IgG-suíno conjugado ao isotiocianato de fluoresceína. A análise dos fatores de risco foi realizada por meio da aplicação de questionários constituídos por perguntas objetivas referentes ao criador, às características gerais da propriedade, ao manejo produtivo, reprodutivo e sanitário. Determinou-se uma prevalência de 26,9\% (92/342) de suínos soropositivos. O fator associado à infecção foi a introdução de reprodutores nas granjas nos últimos cinco anos $(p=0,014$; $O R=1,83$; 
IC=1,13-2,96). Concluiu-se que a infecção por Toxoplasma gondii encontra-se disseminada em suínos criados em granjas tecnificadas no Estado de Alagoas, Brasil. Recomenda-se o controle da população de gatos, a realização de práticas de limpeza e higienização das instalações e realização de testes laboratoriais para diagnóstico da infecção por T. gondii nos animais a serem introduzidos no plantel como medidas de redução dos índices de infecção na região estudada.

TERMOS DE INDEXAÇÃO: Infecção por Toxoplasma gondii, suínos.

\section{INTRODUÇÃO}

Os levantamentos sorológicos relacionados à prevalência da toxoplasmose em rebanhos suínos e outras espécies de produção são importantes tanto para determinar a ocorrência da enfermidade no local estudado como para fornecer dados epidemiológicos que auxiliem na prevenção e controle da infecção em humanos e animais (Fialho \& Araújo 2003).

A infecção nos suínos por Toxoplasma gondii ocorre por meio da ingestão de água, ração ou outros alimentos contaminados por fezes de felinos que contenham oocistos. Para Giraldi et al. (1996), a ingestão de roedores infectados ou de carne infectada, oferecida na forma de restos alimentares, também são responsáveis pela infecção nesta espécie. A transmissão congênita pode ocorrer através da infecção de fêmeas gestantes que não possuem imunidade prévia contra o agente (Weigel et al. 1995).

Para Vidotto et al.(1987), fêmeas suínas gestantes infectadas experimentalmente apresentaram febre, prostração e anorexia. Porém os principais problemas observados estão relacionados às perdas reprodutivas, como repetição de estro, aumento no número de natimortos, mumificados e abortos.

A idade, o grau de higienização e o sistema de produção foram citados como fatores de risco envolvidos na infecção por T. gondii em suínos na Itália (Vilari et al. 2009). O sexo e o sistema de produção foram associados à infecção em suínos no Vietnam (Huong et al. 2007). No Brasil, o grau de tecnificação foi citado como fator associado à infecção no Estado de São Paulo (Oliveira et al. 2007). O sistema de confinamento e o pouco tempo de permanência dos suínos nas granjas reduzem as chances de contato com gatos e roedores, sendo associado à baixa frequência de soropositivos em suínos abatidos em matadouros de Minas Gerais e São Paulo (Pezerico et al. 2007).

Não existem dados sobre os aspectos soroepidemiológicos da infecção por $T$. gondii em granjas suinícolas no Nordeste do Brasil. Sendo assim, objetivou-se com este estudo calcular a prevalência e identificar os fatores de risco associados à infecção por Toxoplasma gondii em suínos criados em granjas tecnificadas no Estado de Alagoas.

\section{MATERIAL E MÉTODOS}

O experimento utilizou animais criados em granjas suinícolas tecnificadas localizadas no Estado de Alagoas, Brasil. Este
Estado encontra-se situado na porção Centro-Oriental do nordeste Brasileiro, pertencente a uma faixa intertropical. Apresenta longos períodos de irradiação solar com variação de 2200 a 2600 horas de sol distribuídas durante o ano. Sendo assim, apresenta temperatura elevada durante a maior parte do ano, com variações ocorrendo entre $22^{\circ} \mathrm{C}$ e $28^{\circ} \mathrm{C}$. O Estado de Alagoas apresenta-se subdividido em três mesorregiões: Leste Alagoano, Agreste Alagoano e Sertão Alagoano (Assis et al. 2007), das quais apenas as duas primeiras foram abordadas na presente pesquisa. Das sete granjas participantes da pesquisa, quatro situavam-se no Leste Alagoano e três no Agreste Alagoano.

O experimento foi realizado entre os meses de janeiro e novembro de 2008. Para compor a amostra do estudo de prevalência considerou-se uma prevalência esperada de 25,5\% (Millar et al. 2008) com nível de confiança de 95\% e erro estatístico de 5\% (Thrusfield 2004), o que determinou uma amostra mínima de 292 animais.

Foram utilizados 342 suínos, sendo 312 matrizes e 30 reprodutores, criados sob condições de tecnificação, oriundos de sete granjas de ciclo completo, distribuídas entre cinco municípios do Estado de Alagoas-Brasil. Os animais eram híbridos, pertencentes a diferentes linhagens genéticas e fornecidos por diferentes empresas que atuam no ramo de melhoramento genético da suinocultura brasileira. As fêmeas eram mantidas sob condições de confinamento total, alojadas em gaiolas, durante a fase de lactação, e em baias coletivas e/ou gaiolas, durante a fase de gestação. Recebiam alimentação balanceada e eram submetidas a manejo de monta natural e/ou inseminação artificial, de acordo com os manejos reprodutivos e sanitários realizados nas diferentes granjas. Da mesma forma, os varrões eram mantidos em baias individuais, sob as mesmas condições de tecnificação disponibilizadas às matrizes.

As amostras de sangue foram colhidas por meio de punção da veia cava cranial e, após centrifugação, as amostras de soro sangüíneo foram acondicionadas em tubos de polipropileno e armazenadas a $-20^{\circ} \mathrm{C}$, para posterior análise laboratorial. O exame sorológico foi realizado no Laboratório de Doenças Infecciosas da UFRPE, onde, para a pesquisa de anticorpos anti-Toxoplasma gondii, empregou-se a técnica de Imunofluorescência Indireta (RIFI), utilizando-se anticorpos anti-IgG-suíno conjugado ao isotiocianato de fluoresceína. Em todas as reações foram incluídos soros padrões positivo e negativo previamente conhecidos. Foi considerado como reação positiva os soros que apresentaram fluorescência total na diluição 1:64. As amostras de soro dos suínos reagentes foram submetidas a diluições seriadas de razão dois até a obtenção da maior diluição positiva na RIFI. O título do soro foi a recíproca da maior diluição que apresentou resultado positivo (Mainar et al. 1996).

A análise dos fatores de risco foi realizada por meio da aplicação de um questionário investigativo, constituído por perguntas objetivas referentes ao criador, às características gerais da propriedade, ao manejo produtivo, reprodutivo e sanitário.

Para identificar os fatores de risco associados à infecção por Toxoplasma gondii, foi realizada análise univariada das variáveis de interesse através do teste qui-quadrado de Pearson ou Exato de Fisher, quando necessário, com nível de significância adotado de 5\%. Posteriormente foi feita uma análise multivariada através do modelo de regressão logística considerando como variável dependente o status sorológico do animal (positivo ou negativo) para T. gondii. As variáveis inde- 
pendentes ou explanatórias consideradas no modelo foram aquelas que apresentaram significância estatística $<0,20$. Essa probabilidade foi estipulada para que possíveis fatores de risco do evento não fossem excluídos da análise (Hosmer \& Lemeshow 1989). O programa SPSS for Windows, versão 12,0 - Statistical Package for the Social Science foi utilizado para a realização dos cálculos estatísticos.

\section{RESULTADOS}

Detectou-se uma prevalência de 26,9\% (92/342) de suínos soropositivos para a infecção por Toxoplasma gondii. Foram observados suínos soropositivos em $85,6 \%$ das granjas estudadas, com variação entre $0 \%$ e $47,9 \%$. Observou-se maior frequência de matrizes soropositivas $(27,6 \%)$ contra $20,0 \%$ para os varrões, não sendo demonstrada associação significativa entre esse fator e a infecção por esse parasito (Quadro 1).

Maior frequência de soropositivos $(33,8 \%)$ foi detectada em animais criados em plantéis com número de matrizes variando entre 100 e 300 , contra $26,2 \%$ de soropositivos nos plantéis com mais de 300 matrizes. A menor frequência de soropositivos (18,6\%) foi observada em animais criados em plantéis inferiores a 100 matrizes (Quadro 1). Estes dados também confirmaram uma associação significativa entre o tamanho dos plantéis e o risco de

\section{Quadro 1. Análise univariada para os fatores de risco associados ou não à infecção por Toxoplasma gondii em suínos criados em granjas tecnificadas no Estado de Alagoas, 2008}

\begin{tabular}{|c|c|c|c|c|}
\hline \multirow[t]{2}{*}{ Variável } & \multirow[t]{2}{*}{$\mathrm{N}$} & \multirow{2}{*}{$\begin{array}{l}\text { RIFI } \\
\text { n (\%) }\end{array}$} & \multicolumn{2}{|c|}{ Análise Univariada } \\
\hline & & & OR (IC 95\%) & $\mathrm{P}$ \\
\hline \multicolumn{5}{|l|}{ Sexo } \\
\hline Macho & 30 & $6(20,0)$ & 1,0 & 0,372 \\
\hline Fêmea & 312 & $86(27,6)$ & $1,52(0,58-4,71)$ & \\
\hline \multicolumn{5}{|l|}{ № de matrizes (cabeças) } \\
\hline$<100$ animais & 113 & $21(18,6)$ & 1,0 & $0,023^{*}$ \\
\hline Entre 100 e 300 & 145 & $49(33,8)$ & $2,24(1,20-4,24)$ & \\
\hline Acima de 300 & 84 & $22(26,2)$ & $1,55(0,74-3,25)$ & \\
\hline \multicolumn{5}{|l|}{ Realiza quarentena } \\
\hline Não & 46 & $7(15,2)$ & 1,0 & 0,055 \\
\hline Sim & 296 & $85(28,7)$ & $2,24(0,95-6,17)$ & \\
\hline \multicolumn{5}{|c|}{ Realiza vazio sanitário (dias) } \\
\hline 4 dias & 191 & $47(24,6)$ & 1,0 & 0,282 \\
\hline$>5$ dias & 151 & $45(29,8)$ & $1,30(0,78-2,16)$ & \\
\hline \multicolumn{5}{|c|}{ Bebedouros comuns para jovens e adultos } \\
\hline Não & 275 & $78(28,4)$ & 1,0 & 0,217 \\
\hline Sim & 67 & $14(20,1)$ & $0,67(0,32 ; 1,31)$ & \\
\hline \multicolumn{5}{|l|}{ Manejo reprodutivo } \\
\hline Monta natural & 113 & $21(18,6)$ & 1,0 & $0,023^{*}$ \\
\hline Inseminação artificial & 84 & $22(26,2)$ & $1,55(0,74 ; 3,25)$ & \\
\hline Monta + inseminação & 145 & $49(33,8)$ & $2,24(1,20 ; 4,24)$ & \\
\hline \multicolumn{5}{|l|}{ Utiliza sêmen refrigerado } \\
\hline Não & 113 & $21(18,6)$ & 1,0 & $0,014^{*}$ \\
\hline Sim & 229 & $71(31,0)$ & $1,97(1,11 ; 3,60)$ & \\
\hline \multicolumn{5}{|c|}{ Introduziu reprodutores no rebanho nos últimos 5 anos } \\
\hline Não & 197 & $43(21,8)$ & 1,0 & $0,014^{*}$ \\
\hline Sim & 145 & $49(33,8)$ & $1,83(1,13-2,96)$ & \\
\hline
\end{tabular}

OR = "Odds ratio"; IC = Intervalo de confiança de 95\%; RIFI = Reação de imunofluorescência. infecção( $p=0,023)$ na análise univariada, sendo, porém, excluído na análise multivariada.

Observou-se maior frequência de soropositivos (33,8\%) nos suínos criados em granjas que utilizavam a monta natural $(\mathrm{MN})$ associada à inseminação artificial $(\mathrm{IA})$, contra $26,2 \%$ de soropositivos nos animais manejados apenas com a IA. A menor frequência $(18,6 \%)$ foi detectada nas granjas que utilizavam apenas a MN. Apesar da associação significativa $(\mathrm{p}=0,023)$ detectada na análise univariada, não houve a confirmação desta variável na análise multivariada. Da mesma forma, observou-se maior frequência de positivos $(31,0 \%)$ em granjas que utilizavam sêmen refrigerado, contra $18,6 \%$ de soropositivos nas granjas que não utilizavam. Apesar da associação significativa detectada $(p=0,014)$, esta variável também foi excluída da análise multivariada.

Maior frequência de soropositivos (27,5\%) foi observada em suínos criados em granjas que possuíam gatos, contra $16,7 \%$ de soropositivos para suínos criados em propriedades que não possuíam gatos. Contudo, não se observou associação significativa entre esta variável e o índice de infecção (Quadro 2). Da mesma forma, o acesso dos gatos aos bebedouros ou comedouros, assim como ao depósito de ração presente nas instalações das granjas e o tipo de alimentação destes felinos, não foram fatores associados à infecção por T. gondii.

Observou-se maior frequência de suínos soropositivos $(33,8 \%)$ quando estes eram criados em granjas que realizaram a introdução de varrões no rebanho nos últimos cinco anos, contra $21,8 \%$ de soropositivos em granjas que não realizaram este manejo. Estes resultados apresentaram uma associação significativa $(p=0,014)$ na análise multivariada, onde os suínos criados em granjas que realizaram a introdução de varrões têm 1,83 vezes mais

Quadro 2. Análise univariada para os fatores de risco associados ou não à infecção por Toxoplasma gondii em suínos criados em granjas tecnificadas no Estado de Alagoas, 2008

\begin{tabular}{|c|c|c|c|c|}
\hline \multirow[t]{2}{*}{ Variável } & \multirow[t]{2}{*}{$\mathrm{N}$} & \multirow{2}{*}{$\begin{array}{l}\text { RIFI } \\
\mathrm{n}(\%)\end{array}$} & \multicolumn{2}{|c|}{ Análise Univariada } \\
\hline & & & OR (I.C.95\%) & $\mathrm{P}$ \\
\hline \multicolumn{5}{|c|}{ Presença de gatos na propriedade } \\
\hline Não & 18 & $3(16,7)$ & 1,0 & 0,419 \\
\hline Sim & 324 & $89(27,5)$ & $1,89(0,53-6,69)$ & \\
\hline \multicolumn{5}{|c|}{ Alimentação dos gatos } \\
\hline Ração & 9 & $0(0,0)$ & & 0,120 \\
\hline Vísceras & 333 & $92(27,6)$ & - & \\
\hline \multicolumn{5}{|c|}{ Acesso dos gatos a água fornecida aos animais } \\
\hline Não & 296 & $85(28,7)$ & 1,0 & 0,055 \\
\hline $\operatorname{Sim}$ & 46 & $7(15,2)$ & $0,45(0,16-1,06)$ & \\
\hline \multicolumn{5}{|c|}{ Acesso dos gatos em depósito ou fábrica de ração } \\
\hline Não & 67 & $14(20,9)$ & 1,0 & 0,217 \\
\hline Sim & 275 & $78(28,4)$ & $1,50(0,76-3,10)$ & \\
\hline \multicolumn{5}{|c|}{ Gatos se alimentam de restos placentários } \\
\hline Não & 44 & $10(22,7)$ & 1 & 0,504 \\
\hline Sim & 298 & $82(27,5)$ & $1,29(0,59-3,06)$ & \\
\hline
\end{tabular}

OR = "Odds ratio" (razão de chances); IC = Intervalo de confiança de $95 \%$. 


\begin{tabular}{|c|c|c|c|c|c|c|}
\hline \multirow[t]{2}{*}{ Dados reprodutivos } & \multicolumn{2}{|c|}{ Negativo } & \multirow{2}{*}{\multicolumn{2}{|c|}{$\begin{array}{l}\text { Positivo } \\
\text { F.A F.R } \\
(\%)\end{array}$}} & \multirow{2}{*}{\begin{tabular}{l}
\multicolumn{1}{c}{ Total } \\
F.A F.R. \\
$(\%)$
\end{tabular}} & \multirow{2}{*}{$\begin{array}{l}\text { Valor } \\
\text { de } p\end{array}$} \\
\hline & $\begin{array}{l}\text { F.A } \\
(\%)\end{array}$ & F.R & & & & \\
\hline \multicolumn{7}{|l|}{ Número de partos } \\
\hline 1 & 14 & 63,6 & 8 & 36,4 & 22100,0 & 0,388 \\
\hline Entre 2 e 3 & 154 & 74,8 & 52 & 25,2 & 206100,0 & \\
\hline$>3$ & 58 & 69,0 & 26 & 31,0 & 84100,0 & \\
\hline \multicolumn{7}{|l|}{ Repetição de cio } \\
\hline Sem repetição & 136 & 71,6 & 54 & 28,4 & 190100,0 & $<0,001^{*}$ \\
\hline $1-2$ & 90 & 73,8 & 32 & 26,2 & 122100,0 & \\
\hline \multicolumn{7}{|l|}{ Aborto } \\
\hline Sem aborto & 218 & 72,7 & 82 & 27,3 & 300100,0 & 0,431 \\
\hline 1 & 8 & 66,7 & 4 & 33,3 & 12100,0 & \\
\hline \multicolumn{7}{|c|}{ Média de nascimento total } \\
\hline$<8$ & 17 & 89,5 & 2 & 10,5 & 19100,0 & 0,115 \\
\hline Entre 8 e 12 & 167 & 69,9 & 72 & 30,1 & 239100,0 & \\
\hline$>12$ & 42 & 77,8 & 12 & 22,2 & 54100,0 & \\
\hline \multicolumn{7}{|l|}{ Média de nascido vivo } \\
\hline$<8$ & 18 & 78,3 & 5 & 21,7 & 23100,0 & 0,779 \\
\hline Entre 8 e 11 & 156 & 71,6 & 62 & 28,4 & 218100,0 & \\
\hline$>11$ & 52 & 73,2 & 19 & 26,8 & 71100,0 & \\
\hline \multicolumn{7}{|l|}{ Natimorto } \\
\hline Não & 76 & 77,6 & 22 & 22,4 & 98100,0 & 0,171 \\
\hline Sim & 150 & 70,1 & 64 & 29,9 & 214100,0 & \\
\hline \multicolumn{7}{|l|}{ Mumificado } \\
\hline Não & 145 & 71,4 & 58 & 28,6 & 203100,0 & 0,587 \\
\hline Sim & 81 & 74,3 & 28 & 25,7 & 109100,0 & \\
\hline \multicolumn{7}{|l|}{ Média de desmame } \\
\hline$<8$ & 48 & 80,0 & 12 & 20,0 & 60100,0 & 0,152 \\
\hline Entre 8 e 10 & 142 & 68,9 & 64 & 31,1 & 206100,0 & \\
\hline \multirow{2}{*}{\multicolumn{7}{|c|}{ Média IDE }} \\
\hline & & & & & & \\
\hline$<5$ & 2 & 50,0 & 2 & 50,0 & 4100,0 & 0,552 \\
\hline Entre 5 e 8 & 139 & 73,5 & 50 & 26,5 & 189100,0 & \\
\hline$>8$ & 85 & 71,4 & 34 & 28,6 & 119100,0 & \\
\hline
\end{tabular}

F.A. = Frequência Absoluta; F.R. $=$ Frequência Relativa; *Associação significativa.

chances de se infectarem do que os animais criados em granjas que não introduziram varrões.

Não se observou associação entre a infecção por $T$. gondii e o desempenho reprodutivo das matrizes participantes desta pesquisa (Quadro 3).

\section{DISCUSSÃO}

Este é o primeiro relato sobre os aspectos epidemiológicos da infecção por Toxoplasma gondii em matrizes e reprodutores suínos criados em granjas suinícolas tecnificadas na região Nordeste do Brasil.

A prevalência de $26,9 \%$ de animais soropositivos observada neste estudo é semelhante a outros estudos realizados em diferentes regiões do Brasil, como os descritos por Garcia et al. (1999) com 24\% e Millar et al. (2008) com 25,5\%, ambos no Estado do Paraná. Caporali et al. (2005) demonstraram freqüências de $0,8 \%$ e $4,7 \%$ para suínos terminados em granjas de São Paulo e Pernambuco, respectivamente. Oliveira et al. (2007) relataram uma prevalência de $20,11 \%$ de soropositivos em suínos criados de forma rústica na microrregião de Registro, também em São
Paulo. Fialho \& Araújo et al. (2003) detectaram frequência de $33,75 \%$ de positivos em suínos abatidos na Grande Porto Alegre.

A variação na frequência de soropositivos entre as granjas ( $0 \%$ a $47,9 \%$ ) corrobora com o descrito anteriormente por Matos et al. (1999) no Estado de Goiás, que também obtiveram variação entre 8,33\% e 60\% nas 40 granjas avaliadas. Da mesma forma, Gamble et al. (1999) detectaram frequência de soropositivos variando de 4\% a 100\% em 85 granjas de cinco Estados dos EUA. Esta variação pode ocorrer principalmente pela variedade de fatores de risco presentes entre regiões ou em granjas de uma mesma região (Suaréz-Aranda et al. 2003, Caporali et al. 2005). Outros fatores também podem favorecer a variação destes resultados, como a presença de felinos e roedores nas propriedades (Dubey et al. 1995), a raça ou a genética dos suínos (Garcia et al. 1999), o tipo de teste diagnóstico utilizado e o título mínimo a ser considerado como positivo (Caporali et al. 2005), assim como a categoria de animais avaliados (Huong 2007), o manejo sanitário e sistema de produção utilizado (Vilari et al. 2009).

Os dados epidemiológicos obtidos nesse estudo são importantes e auxiliam nas ações de prevenção e controle da infecção por T.gondii na população estudada. $\mathrm{O}$ alto índice de animais e rebanhos positivos assume importância na saúde do consumidor, pois a toxoplasmose é uma zoonose de impacto mundial e a infecção nos humanos também pode ocorrer pelo consumo de carne crua ou mal cozida (Caporali et al. 2005). Para Dubey (2008), a falta de medidas que direcionem a redução dos riscos de infecção humana está na ausência de testes diagnósticos individuais nos suínos abatidos para o consumo, principalmente em animais oriundos de áreas endêmicas.

O maior risco de infecção associado à introdução de reprodutores de reposição nos plantéis $(O R=1,83)$ é um aspecto epidemiológico importante relacionado à infecção. Os animais podem ter sido infectados ainda nas granjas multiplicadoras que comercializam animais de alta genética. Outro fator que pode ser considerado é a falha na realização do manejo de quarentena, pois na avaliação dos questionários epidemiológicos realizados nas granjas observou-se que nenhuma delas utilizava a sorologia como método diagnóstico preventivo de doenças antes da introdução de animais adquiridos nos plantéis. Isto pode ter influenciado sobre a maior frequência de soropositivos $(28,7 \%)$ em suínos criados em granjas que realizavam este manejo.

A participação destes reprodutores como disseminadores de T. gondii através da MN ou da IA nos plantéis estudados merece estudos mais aprofundados. Pesquisas sobre este tema ainda não confirmaram a transmissão venérea ou via sêmen do agente para as matrizes suínas apesar de sua detecção no sêmen ou trato genital de varrões. Moura et al. (2007) demonstraram o isolamento de $T$. gondii no sêmen de varrões infectados experimentalmente com oocistos por via oral por períodos de até 56 dias após a infecção, assim como a detecção do agente 
no trato genital (epidídimo e glândula vesicular), sugerindo a possibilidade de transmissão venérea.

Apesar da ausência de associação significativa entre a presença de gatos nas instalações e a infecção dos suínos participantes deste estudo, os questionários epidemiológicos revelaram que $94,7 \%$ das matrizes e reprodutores eram criados em propriedades com presença de gatos, sugerindo uma participação destes animais na infecção dos suínos. Em algumas granjas era visível a presença de grande número de felinos nas instalações, inclusive consumindo restos placentários. Para Hill \& Dubey (2002), os gatos podem eliminar milhões de oocistos pelas fezes após a ingestão de apenas um bradizoíto contido em cisto tecidual de um animal infectado. A grande maioria das pesquisas considera que os oocistos presentes nas fezes destes felinos sejam os responsáveis pela contaminação da água, ração e instalações, constituindo-se em importantes disseminadores do agente nos rebanhos suinícolas (Assadi-Rad et al. 1995, Dubey et al. 1995 Weigell et al. 1995, Lehmann et al. 2003).

Diante da observação da presença dos gatos nas granjas devem ser estimuladas medidas de controle da toxoplasmose suína, principalmente no que se refere à circulação de felinos nas fábricas de ração, monitoramento da água oferecida aos animais, além da limpeza e desinfecção das baias, comedouros e bebedouros. Outro fato importante é a correta realização da quarentena e de testes de diagnóstico para doenças de destaque na suinocultura, incluindo nesse protocolo a toxoplasmose.

A maior frequência de soropositivos nos plantéis com mais de $\mathbf{3 0 0}$ matrizes pode ter sido influenciada por fatores relacionados à maior exigência de tecnificação das granjas. Para Vidotto et al. (1990), estas granjas, na busca por redução de custos, possuem fábricas de ração em suas instalações, o que favorece o maior acúmulo de grãos e cereais em depósitos. Dessa forma, possuem maiores atrativos para roedores e felinos, reservatórios de $T$. gondii. Porém, alguns autores associam o número reduzido de animais no plantel ao baixo nível de tecnificação das granjas e às falhas no manejo sanitário, indicando-os como facilitadores da infecção. Vilari et al. (2009) observaram maior índice de infecção em granjas com menos de 50 animais, contra as que possuíam mais de 50 suínos $(\mathrm{OR}=6,8)$. Assadi-Rad et al. (1995) também observaram maior chance de infecção $(\mathrm{OR}=4,5)$ em granjas com menos de 29 matrizes no plantel.

Possivelmente o estado imunológico das matrizes participantes da pesquisa, estimulado por um contato prévio com $T$. gondii, tenha favorecido a ausência de associação entre a infecção e a ocorrência de falhas reprodutivas. A grande maioria das matrizes $(92,9 \%)$ apresentava mais de dois partos, o que significa uma permanência mínima de um ano nas instalações das granjas, computando-se o período entre a introdução no plantel, as duas gestações e lactações que juntamente com os intervalos para novas inseminações promoveram maior chance de contato com o agente. Para Dubey et al. (1990), as perdas fetais promovidas pela trans- missão congênita podem ocorrer através da infecção de fêmeas gestantes que não possuem imunidade prévia contra o agente. A baixa ingestão de oocistos também pode estar relacionada à ausência de sintomas reprodutivos. Para Moura et al. (2004), a ausência de alterações clínicas e espermáticas em varrões infectados experimentalmente esteve relacionada às baixas doses dos inóculos $\left(1,0 \times 10^{6}\right.$ taquizoítos via subcutânea e $1,5 \times 10^{4}$ oocistos via oral) que foram administrados aos reprodutores.

O momento da infecção das matrizes que dificilmente pode ser diagnosticado em pesquisas de campo pode ter favorecido a ausência de sintomas reprodutivos nas matrizes avaliadas. A maioria das infecções por $T$. gondii nos suínos são subclínicas, com a infecção transplacentária sendo menos comum que a pós-natal. Para Dubey et al. (1990), a ocorrência de fetos mortos e mumificados em duas matrizes infectadas experimentalmente com ração contendo $1,0 \times 10^{3}$ oocistos do T. gondii ocorreu devido à presença do $T$. gondii no trofoblasto que produziu áreas de necrose no corioalantóide e favoreceu a ocorrência de focos de descolamento placentário. Os efeitos reprodutivos promovidos pela infecção ocorreram principalmente devido à presença do agente nas fases iniciais do desenvolvimento fetal.

A frequência de $33,3 \%(4 / 12)$ de matrizes soropositivas apresentando abortos pode ser considerada alta $e$ merece atenção. Embora os abortos sejam pouco freqüentes, podem ocorrer em porcas infectadas durante a gestação (Dubey 1986). Para Kim et al. (2009), a infecção por T.gondii promoveu um surto de aborto em matrizes suínas em granjas da ilha de Jeju na China. A grande maioria dos abortos foi observada na fase inicial da gestação, ocorrendo entre três e cinco dias após a apresentação de sinais clínicos, como febre, anorexia, vômito e prostração.

Diante do exposto, recomenda-se a realização de monitorias sanitárias nas granjas avaliadas quanto à presença de $T$. gondiinos fetos abortados. Para que isso ocorra com sucesso, é importante a logística envolvida na coleta do material para envio aos laboratórios credenciados que realizem o diagnóstico por meio de técnicas de imunohistoquímica, molecular e isolamento, possibilitando a identificação do agente.

Conclui-se que a infecção por Toxoplasma gondii encontra-se disseminada nos rebanhos suínos nas granjas tecnificadas no Estado de Alagoas, Brasil. Indica-se o controle da população de gatos, a realização de práticas de limpeza e higienização das instalações e realização de testes laboratoriais que comprovem a ausência da infecção por T. gondii nos animais a serem introduzidos no plantel, como medidas para reduzir o índice de infecção nas granjas estudadas.

\section{REFERÊNCIAS}

Assadi-rad A.M., New J.C. \& Patton S. 1995. Risk factors associated with transmission of Toxoplasma gondii to sows kept in different management systems in Tennessee. Vet. Parasitol. 57:289-297.

Assis J.S., Alves A.L. \& Nascimento M.C. 2007. Atlas Escolar Alagoas: Espaço Geohistórico e Cultural. Grafset, João Pessoa. 208p. 
Caporali E.H.G., Silva A.V., Mendonça A.O. \& Langoni H. 2005. Comparação de métodos para determinação da prevalência de anticorpos anti-Toxoplasma gondii em suínos dos Estados de São Paulo e Pernambuco, Brasil. Arqs Ciênc. Vet. Zool. 8(1):19-24.

Dubey J.P. 1986. A revew of toxolasmosis in pigs. Vet. Parasitol. 19: 181-223.

Dubey J.P. 2008. The history of Toxoplasma gondii-the first 100 years. J. Eukaryot. Microbiol. 55(6):467-475.

Dubey J.P., Schlfer D.H., Urban Jr J.F. \& Lindsday D.S. 1990. Lesions in fetal pigs with transplacentally-induces toxoplasmosis. Vet. Pathol. 27(6):411-418.

Dubey J.P., Weigel R.M., Seigel A.M., Kitron U.D. \& Mannelli A. 1995. Risck factors for transmission of Toxoplasma gondii on swine farms in Illinois. J. Parasitol. 81(5):736-741.

Fialho C.G. \& Araújo F.A.P. 2003. Detecção de anticorpos para Toxoplasma gondii em soro de suínos criados e abatidos em frigoríficos da região da Grande Porto Alegre-RS, Brasil. Ciência Rural 33(5):893897.

Gamble H.R., Brady R.C. \& Dubey J.P. 1999. Prevalence of Toxoplasma gondii infection in domestic pigs in the New England states. Vet. Parasitol. 82:129-136.

Garcia J.L., Navarro I.T., Ogawa L. \& Oliveira R.C. 1999. Soroprevalência de Toxoplasma gondii, em suínos, bovinos, ovinos e eqüinos, e sua correlação com humanos, felinos e caninos, oriundos de propriedades rurais do Norte do Paraná-Brasil. Ciência Rural 29(1):9197.

Giraldi N., Freire R.L., Navarro I.T., Viotti N.M.A., Bueno S.G. \& Vidotto O. 1996. Estudo da toxoplasmose congênita natural em granjas de suínos em Londrina, PR. Arq. Bras. Med. Vet. Zootec. 48:83-90.

Hill D. \& Dubey J.P. 2002. Toxoplasma gondii: Transmission, diagnosis and prevention. Clin. Microbiol. Infect. 8(10):634-640.

Hosner D.W. \& Lemeshow S. 1989. Applied Logistic Regression. John Wiley and Sons, New York. 241p.

Huong L.T. \& Dubey J.P. 2007. Seroprevalence of Toxoplasma gondii in pigs from Vietnam. J. Parasitol. 93(4):951-952.

Kim J., Kang K., Kang W., Sohn H., Jean Y., Park B.K., Kim Y. \& Kim D. 2009. Porcine abortion outbreak associated with Toxoplasma gondii in Jeju Island, Korea. J. Vet. Sci. 10(2):147-151.

Lehmann T., Graham D.H., Dahl E., Sreekumar C., Launer F., Corn J.L. \& Gamble H.R. 2003. Transmission dynamics of Toxoplasma gondii on a pig farm. Infect. Genet. Evol. 3:135-141.

Mainar R.C., De La Cruz C., Asensio A., Domínguez L. \& VázquezBoland J.A. 1996. Prevalence of agglutinating antibodies to Toxoplasma gondii in small ruminants of the Madrid Region, Spain, and identification of factors influencing seropositivity by multivariate analysis. Vet. Res. Commun. 20(2):153-159.
Matos M.P.C., Miguel M., Moura V.M.B.D., Sobestiansky J. \& Brito L.A.B. 1999. Anticorpos para Toxoplasma gondii em soros de matrizes suínas de granjas que abastecem o mercado consumidor de Goiânia. Hora Vet. 109:9-11.

Millar P.R., Daguer H., Vicente R.T., Costa T., Sobreiro L.G. \& Amendoeira M.R.R. 2008. Toxoplasma gondii: estudo soro-epidemiológico de suínos da região Sudoeste do Estado do Paraná. Pesq. Vet. Bras. 28(1):15-18.

Moura A.B., Filho S.J., Di Mauro D.C., Paim B.B., Pinto F.R. \& Costa A.J. 2004. Avaliação dos parâmetros seminais de cachaços (Sus scrofa) experimentalmente infectados com Toxoplasma gondii. Ciênc. Agrárias 25(2):107-116.

Moura A.B., Costa A.J., Filho S.J., Paim B.B., Pinto F.R. \& Di Mauro D.C. 2007. Toxoplasma gondii in sêmen of experimentally infected swine. Pesq. Vet. Bras. 27(10):430-434.

Oliveira K.R., Domingues P.F., Langoni H., Silva R.C. \& Gottschalk S. 2007. Detecção de anticorpos para Toxoplasma gondii em soros de suínos criados sob condições rústicas na microrregião de Registro, SP, pelo método de aglutinação direta (MAD). Vet. Zootec. 14(2):169175.

Pezerico G.B., Pezerico S.B., Silva R.C., Hoffmann J.F., Camargo L.B. \& Langoni H. 2007. Ocorrência de anticorpos anti-Toxoplasma gondii e anti-Leptospira spp. em suínos abatidos em três abatedouros dos Estados de Minas Gerais e São Paulo. Arqs Inst. Biológico, São Paulo, 74(3):267:270.

Suarez-Aranda F., Galisteu A.J., Hiramoto R.M., Cardoso R.P., Meireles L.R., Miguel O. \& Andrade Jr H.F. 2000. The prevalence and avidity of Toxoplasma gondii lgG antibodies in pigs from Brazil and Peru. Vet. Parasitol. 91(1/2):23-32.

Thrusfield M.V. 2004. Epidemiologia Veterinária. $2^{\mathrm{a}}$ ed. Roca, São Paulo. $556 p$.

Tovi L., Grahama D.H., Dahl E., Sreekumar C., Launer F., Corn J.L., Gamble H.R. \& Dubey J.P. 2003. Transmission dynamics of Toxoplasma gondii on a pig farm. Infect. Genet. Evol. 3:135-141.

Vidotto O., Costa A.J., Balarin M.R.S. \& Rocha M.A. 1987. Toxoplasmose experimental em porcas gestantes. I. Observações clínicas e hematológicas. Arq. Bras. Med. Vet. Zootec. 39(34):623-639.

Vidotto O., Navarro I.T., Giraldi N., Mitsuka R. \& Freire R.L. 1990. Estudos epidemiológicos da toxoplasmose em suínos da região de Londrina, PR. Semina 11(1):53-59.

Villari S., Vesgo G., Petersen E., Cispo A. \& Buffolano W. 2009. Risk factors for toxoplasmosis in pigs bred in Sicily, Southern Italy. Vet. Parasitol. 161(1/2):1-8.

Weigel R.M., Dubey J.P., Seigel A.M., Kitron U.D., Mitchell M.A., Mannelli A., Matheus-Pinilla N.E., Shen S.K., Kwok O.C.H. \& Todd K.S. 1995. Risck factors for transmission of Toxoplasma gondii on swine farms in Illinois. J. Parasitol. 81(5):736-741. 